\title{
Concentric collapse at drug-induced sleep endoscopy: is it really concentric?
}

\author{
Vittorio Rinaldi ${ }^{1}$ (1) $\cdot$ Mario Mantovani ${ }^{2} \cdot$ Manuele Casale $^{1} \cdot$ Lorenzo Pignataro $^{2,3} \cdot$ Marina Carrasco $^{4}$
}

Received: 6 February 2021 / Accepted: 12 February 2021 / Published online: 22 February 2021

(c) The Author(s), under exclusive licence to Springer-Verlag GmbH, DE part of Springer Nature 2021

\section{Dear Editor,}

Retropalatal collapse is one of the most common findings during drug-induced sleep endoscopy (DISE). The main cause of pathological upper airway collapsibility in patients with obstructive sleep apnea (OSA) is impaired pharyngeal neuromuscular tone with increased collapsibility during sleep [1]. The most popular DISE classifications (VOTE, NOHL) define the circumferential retropalatal collapse as "concentric". The term "concentric" commonly should refer to a collapse of $360^{\circ}$ [2]. What we usually observe in patients with retropalatal concentric collapse is an anteroposterior collapse pattern associated with a latero-lateral (transverse) one, which gives an optical effect of a circular $360^{\circ}$ collapse. To be classified as a $360^{\circ}$ collapse, it should theoretically also reveal an anterior collapse of the posterior pharyngeal wall. Although video-stroboscopy exhibited the vibration of the mucosa of the posterior pharyngeal wall during snoring [3], has anyone seen or described a posteroanterior collapse at DISE? No, because this phenomenon is almost impossible under normal conditions. In fact, the posterior pharyngeal wall at the midline is firmly attached to the vertebral bodies through the connections between the buccopharyngeal fascia with the alar fascia and the posterior pharyngeal raphe [4], [5]. Those posterior fascial

Vittorio Rinaldi

rinaldivittorio@yahoo.it

1 Integrated Sleep Surgery Team UCBM, Unit of Otolaryngology-Integrated Therapies in Otolaryngology, Campus Bio-Medico University, Rome, Italy

2 Department of Otolaryngology and Head and Neck Surgery, Fondazione IRCCS Ca' Granda Ospedale Maggiore Policlinico, Milan, Italy

3 Department of Clinical Sciences and Community Health, University of Milan, Milan, Italy

4 Department of Otorhinolaryngology, Doctor Peset University Hospital, Valencia, Spain connections give a remarkable stability to the posterior pharyngeal wall in all sleeping position.

In conclusion, we should remember to take into consideration that when we detect and classify a concentric retropalatal collapse what we are really observing is actually a horseshoe $270^{\circ}$ collapse.

An immediate evidence is that none of the most popular pharyngoplasty techniques addresses the posterior pharyngeal wall, but rather the collapsible soft palate and lateral pharyngeal walls.

Funding No funding was received.

\section{Compliance with ethical standards}

Conflict of interest No conflicts of interest.

\section{References}

1. Askar SM, El-Anwar MW, Quriba AS (2021) Positional awake nasoendoscopic pattern-based surgical decision for correction of retropalatal obstruction in OSA. Eur Arch Otorhinolaryngol. https ://doi.org/10.1007/s00405-020-06559-7

2. da Cunha Viana A Jr, Mendes DL, de Andrade Lemes LN et al (2017) Drug-induced sleep endoscopy in the obstructive sleep apnea: comparison between NOHL and VOTE classifications. Eur Arch Otorhinolaryngol 274:627-635. https://doi.org/10.1007/ s00405-016-4081-7

3. Kotecha B, Kumar G, Sands R, Walden A, Gowers B (2013) Evaluation of upper airway obstruction in snoring patients using digital video stroboscopy. Eur Arch Otorhinolaryngol 270:2141-2147. https://doi.org/10.1007/s00405-013-2370-y

4. Mnatsakanian A, Minutello K, Bordoni B (2020) Anatomy, Head and Neck, Retropharyngeal Space. In: StatPearls, Treasure Island (FL),StatPearls Publishing. Bookshelf ID: NBK537044

5. López-Fernández P, Murillo-González J, Arráez-Aybar LA, de la Cuadra-Blanco C, Moreno-Borreguero A, Mérida-Velasco JR (2019) Early stages of development of the alar fascia (human specimens at 6-12 weeks of development). J Anat 235:1098-1104. https://doi.org/10.1111/joa.13074

Publisher's Note Springer Nature remains neutral with regard to jurisdictional claims in published maps and institutional affiliations. 